\title{
Local criticality close to a quantum Lifshitz point
}

\author{
Mucio A. Continentino ${ }^{a *}$. \\ ${ }^{a}$ Instituto de Física, Universidade Federal Fluminense \\ Campus da Praia Vermelha, Niterói, 24210-340, RJ, Brazil
}

Near quantum Lifshitz points the stiffness of the lifetime of the spin fluctuations is very small. This gives rise to a regime where the critical fluctuations are local in space but extended along the time directions. The Fermi liquid properties in this local quantum regime is governed by a single energy scale, the coherence temperature. These results are relevant for heavy fermions and frustrated systems whenever there is a competition between different instabilities in $q$-space.

Keywords: Heavy fermions; quantum Lifshitz points.

PACS: $71.27 .+\mathrm{a}$

The Gaussian free energy density near a quantum Lifshitz point (QLP) can be written as [1],

$$
\frac{f}{V}=\frac{-3 T}{\pi} \int \frac{d^{d} q}{(2 \pi)^{d}} \int \frac{d \lambda}{e^{\lambda}-1} \tan ^{-1}\left[\frac{\lambda\left(T / T_{c o h}\right)}{1+q^{2} \xi^{2}+q^{4} \xi_{\perp}^{4}}\right]
$$

where the coherence temperature, $k_{B} T_{c o h}=$ $\Gamma_{L} \chi_{L}|\delta|^{\nu z}$ and $|\delta|=\left|J_{Q}-J_{Q}^{c}\right|$ measures the distance to a quantum critical point (QCP) (not to the QLP; this is approached making a stiffness to vanish as discussed below). The crossover exponent takes the value $\nu z=1$ for the problem considered here. The quantity $\Gamma_{L} \chi_{L}$ is defined through the dynamic susceptibility of the local moments, $\chi_{L}(\omega)=\chi_{L}\left(1+i \omega / \Gamma_{L}\right)^{-1}$. Also near the wavevector $Q$ of the incipient antiferromagnetic instability at the QCP, the $q$-dependent interaction is written as, $J_{Q}-J_{Q+q}=A q^{2}+B q^{4}$. For $T<<T_{c o h}$, the free energy can be written as,

$f=-\frac{3 V}{\pi} \frac{T^{2}}{T_{c o h}} \int \frac{d^{d} q}{(2 \pi)^{d}} \int \frac{d \lambda}{e^{\lambda}-1} \frac{\lambda}{1+q^{2} \xi^{2}+q^{4} \xi_{\perp}^{4}}$

which is quadratic in temperature and shows that the coherence temperature marks the onset of Fermi liquid (FL) behavior for $T<<T_{c o h}$. In the equation above, $\xi^{2}=A /|\delta|$ and $\xi_{\perp}^{4}=B /|\delta|$. In the case the stiffness $A \neq 0$, the $q^{4}$-term is irrelevant [2] and we have the usual Gaussian free

*Tel./Fax:+55-21-2620-6735.

E-mail address: mucio@if.uff.br. Work supported by Brazilian Agencies, FAPERJ and CNPq energy of a nearly antiferromagnetic (AF) system in the FL regime [3]. In three dimensions, $d=3$, the free energy can be integrated and we have for the specific heat in the FL regime,

$C / T=\frac{4 \pi^{2}}{\Gamma_{L} \chi_{L} A} \frac{V}{(2 \pi)^{3}} q_{c}\left(1-\frac{\tan ^{-1} q_{c} \xi}{q_{c} \xi}\right)$

where $q_{c} \approx 1 / a$, with $a$ the lattice spacing is a cutoff in momentum space. In the limit $q_{c} \xi>>1$, for $d=3$, this yields,

$C / T=6 \pi^{2} N k_{B}^{2} / A q_{c}^{2}$

which is the usual, non-universal, cut-off dependent result for a $d=3$, nearly AF system. We are interested here in the opposite limit, $q_{c} \xi<<1$ 4. If the stiffness $A$ is small, as is the case close to a QLP, this regime is relevant even close to the $\mathrm{QCP}$, i.e., for $\delta$ small. We find in this limit,

$C / T=\frac{\pi N k_{B}^{2}}{\Gamma_{L} \chi_{L}} \frac{1}{\left|J_{Q}-J_{Q}^{c}\right|}=\frac{\pi N k_{B}}{T_{c o h}}$

which is independent of $A$. In the same regime the uniform susceptibility is given by,

$\chi_{0}=\frac{3 N \mu^{2}}{2 \pi \Gamma_{L} \chi_{L}} \frac{1}{\left|J_{Q}-J_{Q}^{c}\right|}=\frac{3 N \mu^{2}}{2 \pi k_{B} T_{c o h}}$

such that, the Wilson ratio [1],

$\frac{\chi_{0} / \mu^{2}}{C / \pi^{2} k_{B}^{2} T}=\frac{3}{2}=1.5$ 


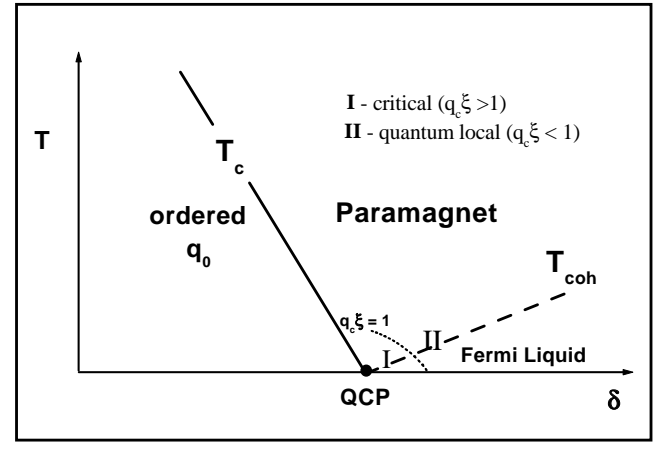

Figure 1. The phase diagram for a heavy fermion system. If the stiffness $A$ is small, the critical region $(I)$ is reduced and the quantum local regime $(I I)$ extends very close to the magnetic quantum critical point.

The electrical resistivity, for $T<<T_{\text {coh }}$ and $q_{c} \xi<<1$, is given by,

$\rho\left(T<<T_{\text {coh }}\right)=\rho_{0} \Gamma_{L} \chi_{L} \frac{\pi^{2}}{3}\left(\frac{T}{T_{\text {coh }}}\right)^{2}=A_{R} T^{2}$

and the Kadowaki-Woods ratio, $A_{R} /(C / T)^{2}$,

$\frac{A_{R}}{(C / T)^{2}}=\frac{\rho_{0} \Gamma_{L} \chi_{L}}{3\left(N k_{B}\right)^{2}}$

is a constant in this regime [1]. The results above could have been obtained, from a local free energy density,

$f_{s f}^{L}=-\frac{3}{\pi} N T \int_{0}^{\infty} \frac{d \lambda}{e^{\lambda}-1} \tan ^{-1}\left(\frac{\lambda T}{T_{c o h}}\right)$

which can be written in the scaling form $f_{s f}^{L}=$ $|g|^{\nu z} F\left[T / T_{\text {coh }}\right]$, such that, $2-\alpha=\nu z$. The hyperscaling relation, $2-\alpha=\nu(d+z)$ [1], in this case is satisfied for an Euclidean dimension $d=0$. This allows to identify the regime $q_{c} \xi<<1$ as a non-trivial quantum local (QL) regime where the critical correlations although being localized in space are extended in the time directions. The effective dimension of the critical fluctuations is consequently, $d_{e f f}=z$. Let us examine in more detail the condition $q_{c} \xi<<1$ or $q_{c} \sqrt{A /|\delta|}<<1$. This implies that, for example, in the $T-|\delta|$ plane there is a crossover line in the non-critical side of the phase diagram, $q_{c} \xi(T)=1$ which separates two regimes: the true critical regime where $q_{c} \xi(T)>>1$ and the quantum local, for $q_{c} \xi(T)<<1$, where correlations are mainly in the time directions. It is interesting that as the stiffness $A \rightarrow 0$, the critical region shrinks and the quantum local regime holds closer and closer to the quantum critical point. The Fermi liquid energy scale in this regime is the coherence temperature, which is independent of the stiffness $A$ and the characteristic time scale is, $\hbar \tau_{\xi}^{-1}=k_{B} T_{\text {coh }}$. Eventually, when $A=0$, the QLP is reached and the critical behavior is governed by the associated fixed point 2]. On the other side of the QLP, for $A<0$, the function $f(q)=A q^{2}+B q^{4}$, vanishes for $q_{0}= \pm \sqrt{|A| / 2 B}$. Near $q_{0}$, we have, $f(q)=-A^{2} / 4 B+(2|A|)\left(q-q_{0}\right)^{2}$. The first term is incorporated in $\delta_{h}=\delta+A^{2} / 4 B$, such that, the correlation length now diverges at the helicoidal transition, $\left|\delta_{h}\right|=0$ [2]. The QLP governs the critical behavior only for $A=0$. $A$ is a relevant perturbation and for any $|A| \neq 0$ the quantum critical behavior is controlled by the usual Hertz fixed point associated with spindensity wave transitions [5]. The zero temperature transition from the paramagnetic to the helicoidal metal is in the same universality class of the para-antiferromagnetic metal transition $(z=2)$. For $d=3$ they are above the critical dimension and are described by a Gaussian theory 11. In the nearly helicoidal metal the correlation length, $\xi_{h}^{2}=2|A| /\left|\delta_{h}\right|$. There is also in this case a crossover line $q_{c} \xi_{h}(T)=1$, such that, for $q_{c} \xi_{h}(T)<1$ a quantum local regime exits where the system presents a similar behavior as in the nearly AF metal.

There are both direct 67 and indirect evidence that heavy fermions and frustrated magnets are close to quantum Lifshitz points. The most dramatic is the heaviness of the quasiparticles which indicates the smallness of the stiffness $A$ (Eq1). How quantum local behavior manifests along the usual non-Fermi liquid path, $|\delta|=0, T \rightarrow 0$ and the role of dangerously irrelevant variables are the subject of present investigations.

We have shown the existence of a significant 


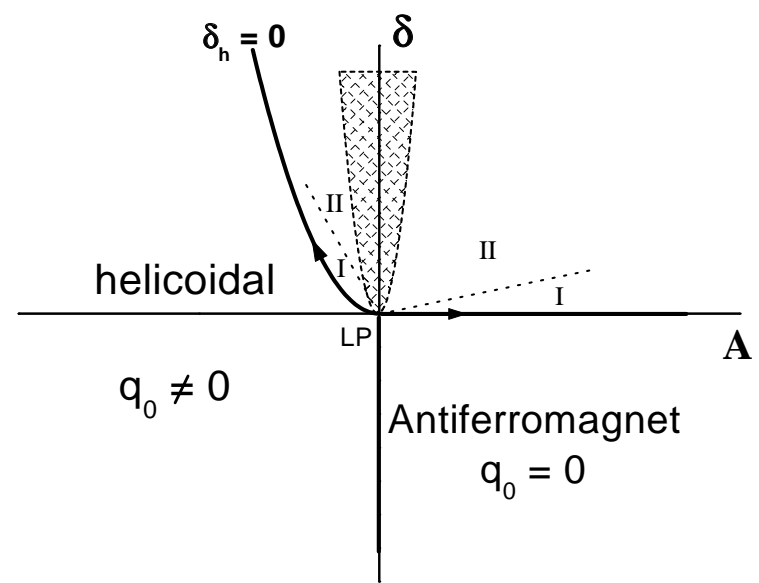

Figure 2. Phase diagram close to a quantum Lifshitz point [2]. In 3d, the lines of para-AF $(|\delta|=0)$ and para-helicoidal $\left(\left|\delta_{h}\right|=0\right)$ phase transitions are governed by quantum critical points with Gaussian exponents and $z=2$. Regions $I$ and $I I$ have the same meaning as in Fig.1. The dashed region is that of Lifshitz quantum criticality 2]. Arrows represent the flow of the renormalization group equations. region in the phase diagram of systems close to a QLP where the physics is governed by fluctuations local in space but extended in time.

\section{REFERENCES}

1. M. A. Continentino, Quantum Scaling in Many Body Systems, World Scientific, Singapore, 2001.

2. R. Ramazashvili, Phys. Rev. B60, 7314 (1999).

3. T. Moriya Spin Fluctuations in Itinerant Electron Magnetism, Springer-Verlag, Berlin, Heidelberg, 1985.

4. M.A.Continentino, Eur. Phys. J. B13, 31 (2000).

5. J. A. Hertz, Phys.Rev. B14, 1165 (1976).

6. A. Schroder et al., Nature, 407, 351 (2000).

7. C. Lacroix et al., Phys.Rev. B54, 15178 (1996). 